\title{
Networked Optimal Control with Random Medium Access Protocol and Packet Dropouts
}

\author{
Chaoqun Zhu, ${ }^{1}$ Ge Guo, ${ }^{2}$ Bin Yang, ${ }^{1}$ and Zhiwen Wang ${ }^{1}$ \\ ${ }^{1}$ School of Electrical and Information Engineering, Lanzhou University of Technology, Lanzhou 730050, China \\ ${ }^{2}$ School of Information Science and Technology, Dalian Maritime University, Dalian 116026, China \\ Correspondence should be addressed to Chaoqun Zhu; zhuchaoqunlut@163.com
}

Received 12 July 2014; Accepted 14 August 2014

Academic Editor: Jun $\mathrm{Hu}$

Copyright (c) 2015 Chaoqun Zhu et al. This is an open access article distributed under the Creative Commons Attribution License, which permits unrestricted use, distribution, and reproduction in any medium, provided the original work is properly cited.

\begin{abstract}
This paper is concerned with an optimal control framework for networked control systems in which the channel access of actuators is governed by a group random access protocol. The system is modeled as a switching Markov jump system with multiple modes according to channel-access status of the actuators, and an independent identical distribution Bernoulli process is used to describe the random packet dropouts of the channel. Then, an optimal control design methodology is addressed to satisfy the quadratic cost function by applying the well-developed theory for jump linear systems and stochastic optimal control while guaranteeing the mean-square exponential stability of networked control systems. And, finally, a numerical example is exploited to demonstrate the effectiveness of the proposed method.
\end{abstract}

\section{Introduction}

Control systems whose components (e.g., sensors, actuators, and controllers) are connected via a real-time communication network are called networked control systems (NCSs). Using a control network has many advantages, such as reduced system wiring and cost, increased flexibility, reliability and safety, easier deployment, diagnosis, and maintenance. However, as any practical communication networks (e.g., wireless networks and underwater acoustic networks) have limited bandwidth, NCSs are subject to several issues that can lead to performance degradation and stability loss. Among these issues, network-induced delays [1-4], packet dropouts [5-8], data rate limitation, and quantization effects [9-11] have attracted the most attention in past decade. This paper is concerned with another interesting problem frequently encountered in NCSs, which is termed medium access constraint. This issue arises when an NCS has a large number of nodes (sensors/actuators or subsystems) while the network has insufficient channels to accommodate them at a time. In such a situation, for a system to meet certain performance requirements, the procedure of controller design should be accompanied by a channel access assignment or scheduling policy for the nodes.

Up to date, there have been generally three types of scheduling policies that are frequently used in addressing the problem of medium access constraint, that is, static scheduling, dynamic scheduling, and hybrid static-dynamic scheduling. Static scheduling policies are also called static access protocols, which are designed offline. One of the earliest efforts on static access scheduling of NCSs can be seen in [12], where the concept of communication sequence was introduced to assign channel access to the nodes. Germinated by this work, a series of network access scheduling methods were presented from different viewpoints; see $[13,14]$, to name a few. Recently, research attention has been paid to dynamic access protocols or dynamic scheduling policies; see, for example, $[15,16]$. A network access protocol is dynamic (or online) when it is designed based on real-time feedback information and the system performance requirement. Generally speaking, static access protocols are quite simple and easily realizable, but they are not adaptable to situations with disturbances or uncertainties (e.g., parameter perturbation). As a result, the 
existing results on static scheduling, like those in $[13,14]$, can only handle NCSs whose dynamics are uncoupled. On the contrary, the dynamic access protocols in $[15,16]$, to mention some, can be used to handle systems with coupled dynamics robustly. However, such online medium access control protocols are either very conservative or computationally complicated. In addition, they require frequent monitoring of the channel for feedback information and complicated processing. One can take advantages of both static and dynamic strategies and derive a combined access control policy, which is called hybrid static-dynamic access protocol. A hybrid policy has been shown to be more promising for managing the constrained network medium access in $[17,18]$.

The scheduling strategy presented in [18] is of special interest because it gives an optimal control solution to address the medium access constraint problem, aside from providing a special type of hybrid static-dynamic access protocol. The problem of integrated optimal control and scheduling was first investigated in [19], where a static scheduling policy was obtained by applying dynamic programming with pruning of the search tree to avoid combinatory explosion. Similar studies along this line can be seen in $[20,21]$. For example, in [20], an optimal static scheduling strategy was derived by solving a complex combinatorial optimization problem with the neighbourhood search method and more efficient search heuristics. In [21], a novel optimal dynamic scheduling framework for NCSs was established on the basis of mixed integer programming and model predictive control techniques. Since network access scheduling and system control are strongly coupled, finding a jointly optimal design approach is indeed very complicated or even unsolvable [22], unless strong assumptions are made on either the plant to be controlled or the network to be scheduled. For this reason, it would be more practical for a control engineer to seek for optimal control solutions for specific medium access protocols that are given. In fact, even for a given medium access policy, the problem of designing an optimal controller is very hard, especially for random access protocols [23].

Though stochastic access protocols are convenient in managing access to shared communication channels without feedback information, they are hardly seen in the literature for control purpose. Here we are interested in investigating optimal control of NCSs in which the network is governed by the random channel-access policy investigated by Wang and Guo [24]. We will consider systems whose actuators share a limited number of channels and the channels are governed by a group random access protocol. At each time, the socalled group random access protocol allows a subset of active actuators to have access to the network and receive the control signal from the controller. At the next time, another subset of actuators is assigned access to the channels. The switching between two successive subsets of active actuators is determined by the random access protocol, which is defined by a vector-valued Markov process [25]. This paper is concerned with an optimal control framework for NCSs in which the channel access of actuators is controlled by a Markov group random access protocol. What makes the problem more complicated is that we consider packet-dropping issue at the same time. The random packet-loss of each network channel is described by an independent identical distribution (iid) Bernoulli process. The channel-access status of the actuators is determined by a group random access protocol, which is described by a Markov process with known transition probability matrix. By transforming the closed-loop system to a Markovian jump system with random packet loss, an optimal control design methodology is presented applying the well-developed theory for jump linear systems and stochastic optimal control.

The organization of the paper is as follows. Section 2 describes the NCS framework and models of access constraints and packet-loss process. The problem of designing an optimal state feedback law is solved in Section 3. Numerical example is presented in Section 4 to show the effectiveness of our results. Finally, Section 5 gives some concluding remarks and discusses future research directions.

Remark 1. It should be pointed out that the issue of control subject to packet dropout and medium access constraint was previously investigated in [26]. An assumption made in this paper is that multiple sensors or actuators in the system share a single unreliable channel that is manipulated by a static access protocol. Compared with [26], this paper considers a more complicated and challenging situation in which the actuators share multiple but insufficient channels according to a group random access protocol. In addition, we have further considered that packet dropouts could randomly occur during packet transmission in the channel.

Notations. The following notations will be used throughout the paper. $R^{n}$ and $R^{m \times n}$ denote the $n$-dimensional Euclidean space and the set of $m \times n$ real matrices, respectively. $C_{m}^{p}$ is the combinatorial number where $p$ elements are selected from a total of $m$ elements. $P>0(\geq 0)$ means that $P$ is real symmetric and positively definite (semidefinite). $X^{T}$ and $X^{-1}$ represent the transpose and the inverse of matrix $X$, and $\operatorname{tr}(X)$ denotes the trace of the matrix $X . \lambda_{\max }(X)$ and $\lambda_{\min }(X)$ are maximal and minimal eigenvalue of the matrix $X$, respectively. $\left\{X_{i}\right\}_{i=1}^{N}$ denotes the set of the matrices $X_{1}, X_{2}, \ldots, X_{N}$. E(v) stands for the mathematical expectation of the stochastic variable $v$, and $\operatorname{Pr}(\cdot)$ means the occurrence probability of the event ".". $Z$ denotes the set of nonnegative integers. Define the sets $T=$ $\{0,1,2, \ldots, T-1\}$ and $N=\{1,2, \ldots, N\}$, where $T$ and $N$ are positive integer.

\section{Problem Formulation}

The NCSs considered in the paper are diagrammatically depicted in Figure 1, where the plant is a linear time-invariant system described by

$$
x(k+1)=A x(k)+B u(k)+G \omega(k),
$$

where $x=\left[x_{1} \cdots x_{n}\right]^{T} \in R^{n}$ is the state vector, $u=\left[u_{1}, \ldots\right.$, $\left.u_{m}\right]^{T} \in R^{m}$ is the control vector actually executed by the actuators, $\omega(k) \in R^{r}$ denotes the white noise with zero means, which satisfies $E\left(\omega(k) \omega(k)^{T}\right)=Z(k)$, and $E\left(\omega(k) \omega(l)^{T}\right)=0$ for all $l \neq k$. $A, B$, and $G$ are constant matrices with appropriate dimensions, respectively. 


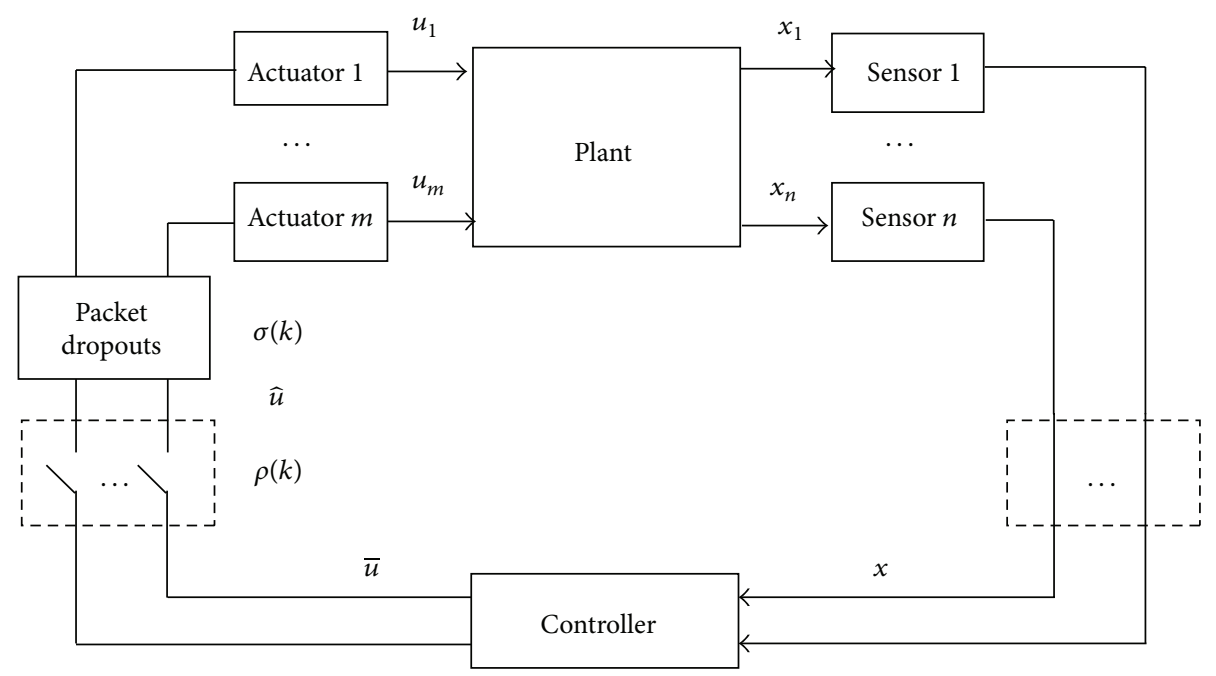

FIGURE 1: A basic NCS configuration.

There are $m$ actuators and $n$ sensors in the NCS. Without loss of generality, we assume that the sensors are time-driven, and the controller and actuators are event-driven. To simplify analysis, we only consider the communication constraints at the actuators side but ignored the same for the sensors. It is assumed that the state of the plant is directly measured by the sensors and sent to the controller with full precision, while the control signals are transmitted in separate packets to the actuators via multiple communication channels. Due to bandwidth limitations, assume that there are $m$ actuators in the system but only $p(<m)$ channels so that the controller can only communicate with $p$ actuators at any time $k$. Therefore, there are $N=C_{m}^{p}$ ( $N$ is a natural number) possible channel-access modes for the actuators. Furthermore, control packet dropouts can randomly occur during transmission due to node failures, network congestion, or communication noise.

For $i=1, \ldots, m$, let the binary-valued function $\rho_{i}(k)$ denote channel-access status of the actuator $i$ at time instant $k$. $\rho_{i}(k)=1$ means that the actuator $i$ is accessing the channel, and the corresponding control signal is transmitted; that is, $u_{i}(k)=\bar{u}_{i}(k)$, where $\bar{u}(k)$ denotes the control vector generated by the controller; otherwise, $\rho_{i}(k)=0$ means that the control input packet is not transmitted and the actuator $i$ will use zero as the control signal; that is, $u_{i}(k)=0$. Then, at time instant $k$, the medium access status of the $m$ actuators is represented by the $m$-to- $p$ communication sequence $\rho(k)=$ $\left[\rho_{1}(k), \rho_{2}(k), \ldots, \rho_{m}(k)\right]^{T}$. Let $M_{\rho}(k)=\operatorname{diag}\{\rho(k)\}$ denote the matrix form of the communication sequence that assigns channel access to the $m$ actuators at time instant $k$. We thus have

$$
\widehat{u}(k)=M_{\rho}(k) \bar{u}(k) .
$$

It is assumed that $M_{\rho}(k)$ could be modeled by a Markov process taking values in a finite set $\left\{M_{\rho}^{i}\right\}_{i=1}^{N}$ with the following conditional probability:

$$
\begin{gathered}
\operatorname{Pr}\left\{M_{\rho}(k+1)=M_{\rho}^{j} \mid M_{\rho}(k)=M_{\rho}^{i}\right\}=\pi_{i j}, \\
\operatorname{Pr}\left\{M_{\rho}(k)=M_{\rho}^{i}\right\}=\pi_{i}(k),
\end{gathered}
$$

where $\pi=\left[\pi_{i j}\right]$ is the transition probability matrix and $\pi_{i j}>0, i, j \in N$, denotes the transition probability from mode $i$ to mode $j$, satisfying $\sum_{j=1}^{N} \pi_{i j}=1 . \pi_{0 i}$ is the initial probability of mode $i$, while $\pi_{i}(k)$ is the probability of mode $i$ at time instant $k$, and $\pi(k)=\left[\begin{array}{llll}\pi_{1}(k) & \pi_{2}(k) & \cdots & \pi_{N}(k)\end{array}\right]^{T}$ obeys the recursion $\pi(k+1)=\pi^{T} \pi(k)$. For simplicity, let $\theta(k)$ be the indicator of the mode of the Markov process, $\theta(k)=i$, if $M_{\rho}(k)=M_{\rho}^{i}$.

For the packet dropout issue, we use a binary-valued random variable $\sigma_{i}(k), i=1, \ldots, m$, to denote the packet-loss phenomenon of the channels at time $k$. When the packet is successfully transmitted to the actuator, we have $\sigma_{i}(k)=1$. If the packet is dropped during transmission, we have $\sigma_{i}(k)=$ 0 . Assume that any packet is lost according to a Bernoulli process with the following probability distribution:

$$
\begin{gathered}
\operatorname{Pr}\left\{\sigma_{i}(k)=1\right\}=E\left\{\sigma_{i}(k)\right\}=\bar{\gamma}_{i}, \\
\operatorname{Pr}\left\{\sigma_{i}(k)=0\right\}=1-E\left\{\sigma_{i}(k)\right\}=1-\bar{\gamma}_{i},
\end{gathered}
$$

where $\bar{\gamma}_{i} \in[0,1]$ is a known constant denoting the $i$ th channel's arrival probability of the control input packet. It is assumed that $\sigma_{i}(k)$ and $\sigma_{i}(l)$ are iid for $k \neq l$ and that different channels are also independent of each other as their expected values could be different; that is, $\bar{\gamma}_{i} \neq \bar{\gamma}_{s}$, for $i \neq s$. In consideration of the effect of packet loss, control signals actually received by the actuators can be expressed as follows:

$$
u(k)=N_{\sigma}(k) \widehat{u}(k),
$$


where $N_{\sigma}(k)=\operatorname{diag}\left\{\sigma_{1}(k), \sigma_{2}(k), \ldots, \sigma_{m}(k)\right\}$ is the probability matrix that denotes the successful transmission of control signals.

We assume that the control network uses a TCP-like protocol with a perfect acknowledgment mechanism. The actuators will send an acknowledgment signal to the controller at time instant $k$, to denote whether the control input packets were successfully delivered to their respective actuators at the previous time instant. It is noteworthy that the actuator acknowledgment has one-step time delay. Then, we define the corresponding information set as $I_{k}=\left\{x(t), \theta(t), N_{\sigma}(t-1) \mid\right.$ $t=0,1, \ldots, k\}$. Apparently, $I_{k} \subset I_{k+1} \subset I_{T}$.

Combining the system dynamics (1), the activation status (2) of the actuators, and the effect of packet dropouts (5) and taking into account the fact that $M_{\rho}^{\theta(k)}$ and $N_{\delta}(k)$ are both diagonal matrices, we can obtain

$$
x(k+1)=A x(k)+B_{\theta(k)} N_{\sigma}(k) \bar{u}(k)+G \omega(k),
$$

where $B_{\theta(k)}=B M_{\rho}^{\theta(k)}$. It is clear that $\left\{B_{\theta(k)} ; k \in Z\right\}$ is also a Markov process.

In the forthcoming development, we will need the following definition.

Definition 2. The NCS is said to be exponentially meansquare stable if, for every initial state $\left(x_{0}, \theta_{0}\right)$, there exist constants $\alpha>0$ and $0<\beta<1$ such that

$$
E\left(\|x(k)\|^{2} \mid x_{0}, \theta_{0}\right) \leq \alpha \beta^{k}\left\|x_{0}\right\|^{2}, \quad \forall k \geq 0 .
$$

The purpose of this paper is to derive an optimal state feedback sequence $\bar{u}(0), \bar{u}(1), \ldots, \bar{u}(T-1)$, for the presented networked system with the initial conditions $\left(x_{0}, \theta_{0}\right)$, such that the system in (6) is exponentially mean-square stable, and minimize the quadratic cost function

$$
\begin{aligned}
& J\left(x_{0}, \theta_{0}, \bar{u}\right) \\
& =E\left(x^{T}(T) Q(T) x(T)\right. \\
& +\sum_{k=0}^{T-1}\left(x^{T}(k) Q(k) x(k)\right. \\
& \left.\left.+\bar{u}^{T}(k) N_{\sigma}(k) R(k) N_{\sigma}(k) \bar{u}(k)\right)\right),
\end{aligned}
$$

where $Q(T) \geq 0, Q(k) \geq 0, R(k)>0, \theta(k) \in N, k \in T$. The minimal is denoted by $J^{*}\left(x_{0}, \theta_{0}\right)$.

Remark 3. There are generally two different strategies to deal with the situation that the actuator does not receive an expected control input packet at a given time. The first one is zero-input control strategy, which denotes the fact that plant's scalar input receives zero values from the designated actuator as its control signal, and the second one is the last available control strategy, which means that the latest control values stored in the actuator buffer will be sent to the plant in this case. In this paper, we adopt the former strategy when actuators could not receive effective control information; see, for example, $[15,27,28]$ and references therein.

Remark 4. From the above description, we know that the system in (6) is a discrete-time Markovian jump linear system with random packet dropouts described by an iid Bernoulli process. This system representation is convenient as it allows us to explore stability analysis and controller design of NCSs by utilizing the well-developed theory for jump linear system and stochastic optimal control.

\section{Control Synthesis with Markov Actuator Assignment and Packet Dropouts}

In this section, we first solve the optimal control problem in finite-time horizon for the system in (6) and derive the optimal control sequence to minimize the quadratic cost function in (8). Subsequently, we will prove that the optimal control sequence can also render the system in (6) exponentially mean-square stable. Define an admissible control input set as $U=\{\bar{u}(k) ; k \in T\}$. By exploiting the independence of the noise input $\omega(k)$, we can know that $x(k), \omega(k), \bar{u}(k)$, and $\omega(k)$ are uncorrelated; then we have

$$
E\left(\omega(k) x^{T}(k)\right)=0, \quad E\left(\omega(k) \bar{u}^{T}(k)\right)=0 .
$$

From the definition of the mathematical expectation, for any measurable functions $g$ and $h$, the following equation holds:

$$
\begin{aligned}
& E\left(g(\omega(k)) h(\theta(k+1)) \mid I_{k}\right) \\
& \quad=E\left(g(\omega(k)) \mid I_{k}\right) \sum_{j=1}^{N} \pi_{\theta(k) j} h(j) .
\end{aligned}
$$

Theorem 5. Given the system in (6), with noise free measurement of the state vector $x(k)$, that is, $\omega(k)=0$, the control law that minimizes the quadratic cost function in (8) is given by

$$
\bar{u}(k)=K_{\theta(k)} x(k)
$$

with the optimal cost function

$$
\begin{aligned}
& J^{*}\left(x_{0}, \theta_{0}\right) \\
&=\sum_{i=1}^{N}\left(\operatorname{tr}\left(\pi_{i}(0) x(0) P_{i}(0) x^{T}(0)\right)\right. \\
& \\
&\left.\quad+\sum_{k=0}^{T-1} \pi_{i}(k) \operatorname{tr}\left(G Z(k) G^{T} \bar{P}_{i}(k+1)\right)\right),
\end{aligned}
$$


where

$$
\begin{aligned}
K_{\theta(k)}(k) & \\
= & -\left(\sum_{I \subset \Omega_{m}} p_{I} N_{I}\left(R(k)+B_{\theta(k)}^{T} \bar{P}_{\theta(k)}(k+1) B_{\theta(k)}\right) N_{I}\right)^{-1} \\
& \times \bar{N} B_{\theta(k)}^{T} \bar{P}_{\theta(k)}(k+1) A,
\end{aligned}
$$

$P_{\theta(k)}(k)$

$$
\begin{aligned}
& =Q(k)+A^{T} \bar{P}_{\theta(k)}(k+1) A \\
& -A^{T} \bar{P}_{\theta(k)}(k+1) B_{\theta(k)} \bar{N} \\
& \times\left(\sum_{I \subset \Omega_{m}} p_{I} N_{I}\left(R(k)+B_{\theta(k)}^{T} \bar{P}_{\theta(k)}(k+1) B_{\theta(k)}\right) N_{I}\right)^{-1} \\
& \times \bar{N} B_{\theta(k)}^{T} \bar{P}_{\theta(k)}(k+1) A, \\
& \bar{P}_{\theta(k)}(k+1)=\sum_{j=1}^{N} \pi_{\theta(k) j} P_{j}(k+1)>0, \\
& P_{\theta(T)}(T)=Q(T),
\end{aligned}
$$

$\theta(k) \in N, \quad \bar{N}=\operatorname{diag}\left\{\bar{\gamma}_{1}, \ldots, \bar{\gamma}_{m}\right\}, \quad k=T-1, \ldots, 1,0$

Proof. Let us define the following optimal value function $V(\theta(k), x(k), k)$ :

$$
\begin{aligned}
& V(\theta(T), x(T), T)=E\left(x^{T}(T) Q(T) x(T) \mid I_{T}\right), \\
& V(\theta(k), x(k), k) \\
& \quad=\min _{\bar{u}(k)} E\left(x^{T}(k) Q(k) x(k)\right. \\
& \quad+\bar{u}^{T}(k) N_{\sigma}(k) R(k) N_{\sigma}(k) \bar{u}(k) \\
& \left.\quad+V(\theta(k+1), x(k+1), k+1) \mid I_{k}\right) .
\end{aligned}
$$

Obviously, $J^{*}\left(x_{0}, \theta_{0}\right)=V(\theta(0), x(0), 0)$. We claim that the optimal value function can be written as follows:

$$
\begin{array}{r}
V(\theta(k), x(k), k)=E\left(x^{T}(k) P_{\theta(k)}(k) x(k) \mid I_{k}\right)+\alpha(k), \\
k=T, \ldots, 1,0,
\end{array}
$$

where $\alpha(T)=0, \alpha(k)=\sum_{t=k}^{T-1} \sum_{i=1}^{N} \pi_{i}(t) \operatorname{tr}\left(G Z(k) G^{T} \bar{P}_{i}(t+1)\right)$, and $k=T-1, \ldots, 1,0$. We will prove the theorem by deducing that (15), (16), and (17) are equivalent. The mathematical induction is employed in the derivation process.
It is easy to verify that the claim in (17) is true for $k=T$ with parameters $P_{\theta(T)}(T)=Q(T), \theta(T) \in N$, and $\alpha(T)=0$. We suppose that the claim in (17) is true for $k+1$ :

$$
\begin{aligned}
& V(\theta(k+1), x(k+1), k+1) \\
& =E\left(x^{T}(k+1) P_{\theta(k+1)}(k+1) x(k+1) \mid I_{k+1}\right) \\
& \quad+\alpha(k+1) .
\end{aligned}
$$

The value function at time $k$ is then

$$
\begin{gathered}
V(\theta(k), x(k), k) \\
=\min _{\bar{u}(k)}\left(E \left(x^{T}(k) Q(k) x(k)\right.\right. \\
+\bar{u}^{T}(k) N_{\sigma}(k) R(k) N_{\sigma}(k) \bar{u}(k) \\
\left.\left.+V(\theta(k+1), x(k+1), k+1) \mid I_{k}\right)\right) \\
=\min _{\bar{u}(k)}\left(E \left(x^{T}(k) Q(k) x(k)\right.\right. \\
\left.+\bar{u}^{T}(k) N_{\sigma}(k) R(k) N_{\sigma}(k) \bar{u}(k) \mid I_{k}\right) \\
+E\left(E\left(x^{T}(k+1) P_{\theta(k+1)}(k+1) x(k+1) \mid I_{k+1}\right)\right. \\
\left.\left.+\alpha(k+1) \mid I_{k}\right)\right) .
\end{gathered}
$$

Note that, the conditional expectations have the smoothing property [29] as follows:

$$
E\left(V(k+1) \mid I_{k}\right)=E\left(E\left(V(k+1) \mid I_{k+1}\right) \mid I_{k}\right) .
$$

Substituting (20) into (19) leads to

$$
\begin{aligned}
& V(\theta(k), x(k), k) \\
& =\min _{\bar{u}(k)}\left(E \left(x^{T}(k) Q(k) x(k)\right.\right. \\
& \left.+\bar{u}^{T}(k) N_{\sigma}(k) R(k) N_{\sigma}(k) \bar{u}(k) \mid I_{k}\right) \\
& +E\left(x^{T}(k+1) P_{\theta(k+1)}(k+1) x(k+1) \mid I_{k}\right) \\
& +\alpha(k+1)) .
\end{aligned}
$$

Since medium access constraints and packet dropouts are two independent random processes, $\theta(k)$ and $N_{\sigma}(k)$ are uncorrelated random variables. According to (6), we can obtain

$$
\begin{aligned}
E\left(x^{T}(k+1) P_{\theta(k+1)}(k+1) x(k+1) \mid I_{k}\right) \\
=E\left(\left(A x(k)+B_{\theta(k)} N_{\sigma}(k) \bar{u}(k)+G \omega(k)\right)^{T}\right. \\
\quad \times P_{\theta(k+1)}(k+1) \\
\left.\quad \times\left(A x(k)+B_{\theta(k)} N_{\sigma}(k) \bar{u}(k)+G \omega(k)\right) \mid I_{k}\right)
\end{aligned}
$$




$$
\begin{aligned}
=E( & x^{T}(k) A^{T} P_{\theta(k+1)}(k+1) A x(k) \\
& +2 \bar{u}^{T}(k) N_{\sigma}(k) B_{\theta(k)}^{T} P_{\theta(k+1)}(k+1) A x(k) \\
& +2 x^{T}(k) A^{T} P_{\theta(k+1)}(k+1) G \omega(k) \\
& +\bar{u}^{T}(k) N_{\sigma}(k) B_{\theta(k)}^{T} P_{\theta(k+1)}(k+1) \\
& \times B_{\theta(k)} N_{\sigma}(k) \bar{u}(k) \\
& +2 \bar{u}^{T} N_{\sigma}(k)(k) B_{\theta(k)}^{T}(k) P_{\theta(k+1)}(k+1) G \omega(k) \\
& \left.+\omega^{T}(k) G^{T} P_{\theta(k+1)}(k+1) G \omega(k) \mid I_{k}\right) .
\end{aligned}
$$

Taking the mathematical expectation on (22) and substituting (10) into (22) yield

$$
\begin{aligned}
E\left(x^{T}(k+1) P_{\theta(k+1)}(k+1) x(k+1) \mid I_{k}\right) \\
=\quad x^{T}(k) A^{T} \bar{P}_{\theta(k)}(k+1) A x(k) \\
+2 \bar{u}^{T}(k) \bar{N} B_{\theta(k)}^{T} \bar{P}_{\theta(k)}(k+1) A x(k) \\
+2 \operatorname{tr}\left(G E\left(\omega(k) x^{T}(k) \mid I_{k}\right) A^{T} \bar{P}_{\theta(k)}(k+1)\right) \\
+2 \bar{N} \operatorname{tr}\left(G E\left(\omega(k) \bar{u}^{T}(k) \mid I_{k}\right) B_{\theta(k)}^{T} \bar{P}_{\theta(k)}(k+1)\right) \\
+\operatorname{tr}\left(G E\left(\omega(k) \omega^{T}(k) \mid I_{k}\right) G^{T} \bar{P}_{\theta(k)}(k+1)\right) \\
+\bar{u}^{T}(k)\left(\sum_{I \subset \Omega_{m}} p_{I} N_{I} B_{\theta(k)}^{T} \bar{P}_{\theta(k)}(k+1) B_{\theta(k)} N_{I}\right) \bar{u}(k),
\end{aligned}
$$

where $I$ is the set of channels for successful transmission data. It is easy to see that $I$ is a subset of the index set $\Omega_{m}=$ $\{1,2, \ldots, m\}$ and has $2^{m}$ possible values. $p_{I}=\prod_{i \in I} \bar{\gamma}_{i} \prod_{i \notin I}(1-$ $\bar{\gamma}_{i}$ ) denotes the probability of $N_{\sigma}(k)$ taking values in $N_{I}$, where $N_{I}$ is a diagonal matrix and its diagonal elements are

$$
\left(N_{I}\right)_{i i}= \begin{cases}1, & \text { if } i \in I \\ 0, & \text { if } i \notin I .\end{cases}
$$

It follows from (9) that

$$
\begin{gathered}
E\left(\operatorname{tr}\left(G E\left(\omega(k) x^{T}(k) \mid I_{k}\right) A^{T} \bar{P}_{\theta(k)}(k+1)\right)\right)=0 \\
E\left(\operatorname{tr}\left(G \bar{N} E\left(\omega(k) \bar{u}^{T}(k) \mid I_{k}\right) B_{\theta(k)}^{T} \bar{P}_{\theta(k)}(k+1)\right)\right)=0 \\
E\left(\operatorname{tr}\left(G E\left(\omega(k) \omega^{T}(k) \mid I_{k}\right) G^{T} \bar{P}_{\theta(k)}(k+1)\right)\right) \\
=E\left(\operatorname{tr}\left(G E\left(\omega(k) \omega^{T}(k)\right) G^{T} \bar{P}_{\theta(k)}(k+1)\right)\right) \\
=\sum_{i=1}^{N} \pi_{i}(k) \operatorname{tr}\left(G Z(k) G^{T} \bar{P}_{i}(k+1)\right)
\end{gathered}
$$

Substituting (25) and (26) into (23), we obtained

$$
\begin{aligned}
E\left(x^{T}(k+1) P_{\theta(k+1)}(k+1) x(k+1) \mid I_{k}\right) \\
=x^{T}(k) A^{T} \bar{P}_{\theta(k)}(k+1) A x(k) \\
\quad+2 \bar{u}^{T}(k) \bar{N} B_{\theta(k)}^{T} \bar{P}_{\theta(k)}(k+1) B_{\theta(k)} A x(k) \\
\quad+\sum_{i=1}^{N} \pi_{i}(k) \operatorname{tr}\left(G Z(k) G^{T} \bar{P}_{i}(k+1)\right) \\
\quad+\bar{u}^{T}(k)\left(\sum_{I \subset \Omega_{m}} p_{I} N_{I} B_{\theta(k)}^{T} \bar{P}_{\theta(k)}(k+1) B_{\theta(k)} N_{I}\right) \bar{u}(k) .
\end{aligned}
$$

Substituting (27) into (21), then one has

$$
\begin{aligned}
V(\theta(k), x( & k), k) \\
=\min _{\bar{u}(k)}( & x^{T}(k) Q(k) x(k) \\
& +x^{T}(k) A^{T} \bar{P}_{\theta(k)}(k+1) A x(k) \\
& +2 \bar{u}^{T}(k) \bar{N} B_{\theta(k)}^{T} \bar{P}_{\theta(k)}(k+1) A x(k)+\alpha(k+1) \\
& +\sum_{i=1}^{N} \pi_{i}(k) \operatorname{tr}\left(G Z(k) G^{T} \bar{P}_{i}(k+1)\right) \\
& +\bar{u}^{T}(k) \\
& \times\left(\sum_{I \subset \Omega_{m}} p_{I} N_{I}\left(R(k)+B_{\theta(k)}^{T} \bar{P}_{\theta(k)}(k+1) B_{\theta(k)}\right) N_{I}\right) \\
& \times \bar{u}(k)) .
\end{aligned}
$$

Since the control input $\bar{u}(k)$ is unconstrained, let

$$
\frac{\partial V(\theta(k), x(k), k)}{\partial \bar{u}(k)}=0
$$

which yields

$$
\begin{aligned}
& 2 \bar{N} B_{\theta(k)}^{T} \bar{P}_{\theta(k)}(k+1) A x(k) \\
& +2\left(\sum_{I \subset \Omega_{m}} p_{I} N_{I}(R(k)\right. \\
& \left.\left.+B_{\theta(k)}^{T} \bar{P}_{\theta(k)}(k+1) B_{\theta(k)}\right) N_{I}\right) \bar{u}(k) \\
& \quad=0 .
\end{aligned}
$$

From $R(k)>0$, we have that $\left(R(k)+B_{\theta(k)}^{T} \bar{P}_{\theta(k)}(k+1)\right.$ $\left.B_{\theta(k)}\right)>0$, so we can obtain the optimal control sequence in 
(11). Noting that $\alpha(k)=\sum_{i=1}^{N} \pi_{i}(k) \operatorname{tr}\left(G Z(k) G^{T} \bar{P}_{i}(k+1)\right)+$ $\alpha(k+1)$ and substituting (11) into the optimal value function $V(\theta(k), x(k), k)$, we obtained

$$
\begin{aligned}
V(\theta(k), & x(k), k) \\
=E( & x^{T}(k)\left(Q(k)+A^{T} \bar{P}_{\theta(k)}(k+1) A\right. \\
& -A^{T} \bar{P}_{\theta(k)}(k+1) B_{\theta(k)}^{T} \bar{N} \\
& \times\left(\sum_{I \subset \Omega_{m}} p_{I} N_{I}\left(R(k)+B_{\theta(k)}^{T} \bar{P}_{\theta(k)}(k+1) B_{\theta(k)}\right) N_{I}\right)^{-1} \\
& \left.\left.\times \bar{N} B_{\theta(k)} \bar{P}_{\theta(k)}(k+1) A\right) x(k) \mid I_{k}\right)+\alpha(k) .
\end{aligned}
$$

Letting

$$
\begin{aligned}
P_{\theta(k)}(k) \\
= \\
\quad-A^{T} \bar{P}_{\theta(k)}(k)+A^{T} \bar{P}_{\theta(k)}(k+1) B_{\theta(k)} \bar{N} \\
\quad \times\left(\sum_{I \subset \Omega_{m}} p_{I} N_{I}\left(R(k)+B_{\theta(k)}^{T} \bar{P}_{\theta(k)}(k+1) B_{\theta(k)}\right) N_{I}\right)^{-1} \\
\quad \times \bar{N} B_{\theta(k)}^{T} \bar{P}_{\theta(k)}(k+1) A,
\end{aligned}
$$

we can obtain

$$
V(\theta(k), x(k), k)=E\left(x^{T}(k) P_{\theta(k)}(k) x(k) \mid I_{k}\right)+\alpha(k) .
$$

From the last equation we can easily show that (17) is satisfied also at time instant $k$ for all $x(k)$ if and only if the matrices $P_{\theta(k)}(k)$ satisfy (14). As a consequence, since $J^{*}\left(x_{0}, \theta_{0}\right)=$ $V(\theta(0), x(0), 0)$, it follows that the optimal value function is

$$
\begin{aligned}
J^{*}\left(x_{0}, \theta_{0}\right)= & E\left(x^{T}(0) P_{\theta(0)}(0) x(0)\right)+\alpha(0) \\
= & \sum_{i=1}^{N}\left[\operatorname{tr}\left(\pi_{i}(0) x(0) P_{i}(0) x^{T}(0)\right)\right. \\
& \left.+\sum_{k=0}^{T-1} \pi_{i}(k) \operatorname{tr}\left(G Z(k) G^{T} \bar{P}_{i}(k+1)\right)\right] .
\end{aligned}
$$

The proof is thus completed.

Remark 6. Note that the problem under consideration is challenging since the channel access of actuators is controlled by a Markov group random access protocol. Furthermore, it is difficult to apply directly stochastic optimal control theory because of packet-dropping issue considered simultaneously.
Even so, we still achieved a useful analysis and controller design technique in Theorem 5 for NCSs under stochastic access protocol and packet-loss.

In the following, we will continue to prove that the optimal control sequence in (11) is not only minimizing the quadratic cost function in (8) but also rendering the system in (6) exponentially mean-square stable.

Theorem 7. If we ignore the effect of the white noise in system (6), that is, $\omega(k)=0, k \in T$, then the optimal control sequence in (11) renders the system in (6) exponentially mean-square stable.

Proof. From (14), we can obtain $P_{\theta(k)}(k)>0, \theta(k) \in N$. Define the Lyapunov function $V(k)=x^{T}(k) P_{\theta(k)} x(k)$, which is positively definite when $x(k)$ is not equivalent to zero. Then

$$
\begin{aligned}
& E\left(V(k+1) \mid I_{k}\right)-V(k) \\
& =E\left(x^{T}(k+1) P_{\theta(k+1)}(k+1) x(k+1) \mid I_{k}\right) \\
& -x^{T}(k) P_{\theta(k)}(k) x(k) \\
& =E\left(\left(A x(k)+B_{\theta(k)} N_{\sigma}(k) \bar{u}(k)\right)^{T}\right. \\
& \left.\times P_{\theta(k+1)}(k+1)\left(A x(k)+B_{\theta(k)} N_{\sigma}(k) \bar{u}(k)\right) \mid I_{k}\right) \\
& -x^{T}(k) P_{\theta(k)}(k) x(k) \\
& =x^{T}(k) A^{T} \bar{P}_{\theta(k)}(k+1) A x(k) \\
& +2 \bar{u}^{T}(k) \bar{N} B_{\theta(k)}^{T} \bar{P}_{\theta(k)}(k+1) B_{\theta(k)} A x(k) \\
& +\bar{u}^{T}(k)\left(\sum_{I \subset \Omega_{m}} p_{I} N_{I} B_{\theta(k)}^{T} \bar{P}_{\theta(k)}(k+1) B_{\theta(k)} N_{I}\right) \bar{u}(k) \\
& -x^{T}(k) P_{\theta(k)}(k) x(k) \\
& =-x^{T}(k)\left(K_{\theta(k)}^{T}\left(\sum_{I \subset \Omega_{m}} p_{I} N_{I} R(k) N_{I}\right) K_{\theta(k)}\right. \\
& +Q(k)) x(k) .
\end{aligned}
$$

Let

$$
F_{\theta(k)}=K_{\theta(k)}^{T}\left(\sum_{I \subset \Omega_{m}} p_{I} N_{I} R(k) N_{I}\right) K_{\theta(k)}+Q(k),
$$

and hence

$$
\begin{aligned}
E\left(V(k+1) \mid I_{k}\right) & =V(k)-x^{T}(k) F_{\theta(k)} x(k) \\
& \leq\left(1-\frac{\lambda_{\min }\left(F_{\theta(k)}\right)}{\lambda_{\max }\left(P_{\theta(k)}\right)}\right) V(k) \\
& <\left(1-\frac{\mu}{\sigma}\right) V(k)=\beta V(k),
\end{aligned}
$$


where $0<\mu<\lambda_{\min }\left(F_{\theta(k)}\right), \sigma>\lambda_{\max }\left(P_{\theta(k)}\right)$. Apparently, $\mu<\quad$ is given by $\sigma, 0<\beta<1$. Similar to (37), we obtain

$$
E\left(V(k) \mid I_{k-1}\right)<\beta V(k-1) .
$$

Applying the conditional expectations to (38) and considering the smoothing property in (20), we obtained

$$
\begin{aligned}
E\left(V(k) \mid I_{k-2}\right) & =E\left(E\left(V(k) \mid I_{k-1}\right) \mid I_{k-2}\right) \\
& <\beta E\left(V(k-1) \mid I_{k-2}\right) \\
& <\beta^{2} V(k-2) .
\end{aligned}
$$

Continuing this process, we finally obtained

$$
E(V(k))<\beta^{k} V(0) .
$$

So

$$
E\left(x^{T}(k) P_{\theta(k)}(k) x(k)\right)<\beta^{k} x_{0}^{T} P_{\theta_{0}}(0) x_{0} .
$$

Then

$$
\begin{aligned}
E\left(x^{T}(k) x(k)\right) & <\frac{\beta^{k}}{\lambda_{\min }\left(P_{\theta(k)}(k)\right)} x_{0}^{T} P_{\theta_{0}} x_{0} \\
& \leq \frac{\lambda_{\max }\left(P_{\theta_{0}}(0)\right)}{\lambda_{\min }\left(P_{\theta(k)}(k)\right)} \beta^{k} x_{0}^{T} x_{0} \\
& =\alpha \beta^{k} x_{0}^{T} x_{0},
\end{aligned}
$$

where $\alpha=\lambda_{\max }\left(P_{\theta_{0}}(0)\right)\left(\lambda_{\min }\left(P_{\theta(k)}(k)\right)\right)^{-1}$. Therefore, the system in (6) is exponentially mean-square stable by Definition 2. This completes the proof.

Remark 8. If there is no packet dropout during data transmission from controller to actuators, the system in (6) can be rewritten as follows:

$$
x(k+1)=A x(k)+B_{\theta(k)} \bar{u}(k)+G \omega(k) .
$$

Apparently, the system in (43) is a canonical Markovian jump linear system. We can derive the following corollaries immediately for NCSs with group random access protocol by using similar lines of proofs of Theorems 5 and 7.

Corollary 9. Given the system in (43), with noise free measurement of the state vector $x(k)$, that is, $\omega(k)=0$, the optimal control sequence that minimizes the quadratic cost function,

$$
\begin{aligned}
& J\left(x_{0}, \theta_{0}, \bar{u}\right) \\
& =E\left(x^{T}(T) Q(T) x(T)\right. \\
& \left.\quad+\sum_{k=0}^{T-1}\left(x^{T}(k) Q(k) x(k)+\bar{u}^{T}(k) R(k) \bar{u}(k)\right)\right),
\end{aligned}
$$

$$
\bar{u}(k)=K_{\theta(k)} x(k),
$$

with the optimal cost function

$$
\begin{aligned}
J^{*}\left(x_{0}, \theta_{0}\right)=\sum_{i=1}^{N}( & \operatorname{tr}\left(\pi_{i}(0) x(0) P_{i}(0) x^{T}(0)\right) \\
& \left.+\sum_{k=0}^{T-1} \pi_{i}(k) \operatorname{tr}\left(G Z(k) G^{T} \bar{P}_{i}(k+1)\right)\right),
\end{aligned}
$$

where

$$
\begin{aligned}
K_{\theta(k)}(k)= & -\left(R(k)+B_{\theta(k)}^{T} \bar{P}_{\theta(k)}(k+1) B_{\theta(k)}\right)^{-1} \\
& \times B_{\theta(k)}^{T} \bar{P}_{\theta(k)}(k+1) A, \\
P_{\theta(k)}(k)= & Q(k)+A^{T} \bar{P}_{\theta(k)}(k+1) A \\
& -A^{T} \bar{P}_{\theta(k)}(k+1) B_{\theta(k)} \\
& \times\left(R(k)+B_{\theta(k)}^{T} \bar{P}_{\theta(k)}(k+1) B_{\theta(k)}\right)^{-1} \\
& \times B_{\theta(k)}^{T} \bar{P}_{\theta(k)}(k+1) A, \\
& P_{\theta(k)}=P_{\theta(k)}^{T}>0, \\
& \bar{P}_{\theta(k)}(k+1)=\sum_{j=1}^{N} \pi_{\theta(k) j} P_{j}(k+1), \\
& P_{\theta(T)}(T)=Q(T), \\
& \theta(k) \in N, \quad k=T-1, \ldots, 1,0 .
\end{aligned}
$$

Corollary 10. If we ignore the effect of the white noise, that is, $\omega(k)=0, k \in T$, then the optimal control sequence in (45) renders the system in (43) exponentially mean-square stable.

\section{Numerical Example}

To illustrate the effectiveness of the presented method, we give a numerical example. Consider a discrete-time plant in the form of (1) with the following parameters:

$$
\begin{gathered}
A=\left[\begin{array}{cc}
0.1600 & -1.2005 \\
-1.1042 & -2.3890
\end{array}\right], \quad B=\left[\begin{array}{cc}
-1.5350 & 1.8918 \\
-1.2902 & -1.6869
\end{array}\right], \\
G=\left[\begin{array}{ll}
0.2000 & 0.0000 \\
0.0000 & 0.2000
\end{array}\right] .
\end{gathered}
$$

The eigenvalues of $A$ are 0.6030 and -2.8320 ; thus the above system is open-loop and unstable. Our objective is to derive an optimal state feedback controller to render the system in (6) exponentially mean-square stable and minimize the quadratic cost function in (8). Assume that packet arrival probability of channels 1 and 2 is $\bar{\gamma}_{1}=0.76$ and $\bar{\gamma}_{2}=0.70$, 


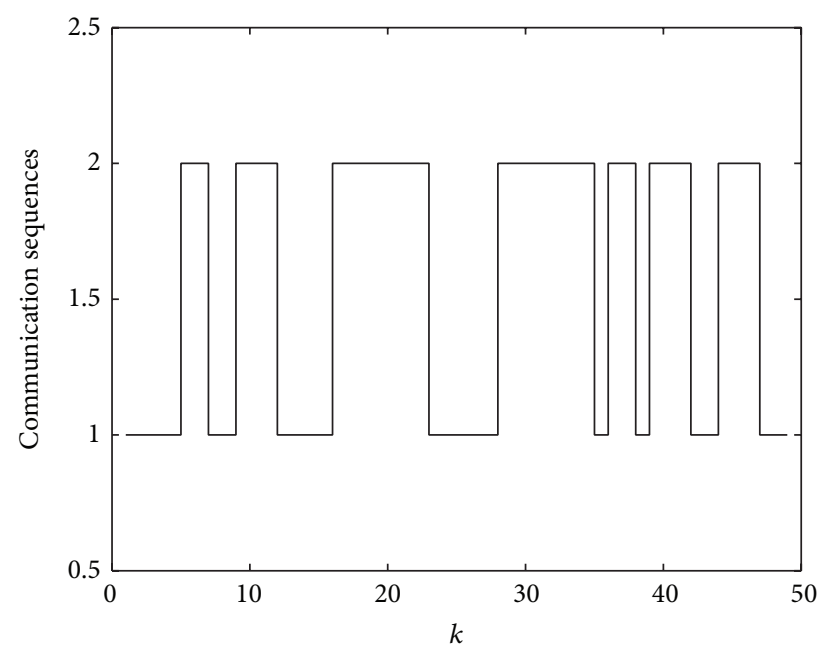

FIGURE 2: Activation mode sequences of actuators.

respectively. We consider the worst-case scenario, where $p=$ 1. Then, actuator assignment sequences matrices are

$$
M_{\rho}^{1}=\operatorname{diag}\{1,0\}, \quad M_{\rho}^{2}=\operatorname{diag}\{0,1\} .
$$

In the simulation, we use the following transition matrix for the Markov chains:

$$
\pi=\left[\begin{array}{ll}
0.6975 & 0.3025 \\
0.3150 & 0.6850
\end{array}\right]
$$

We formulate the optimal control problem with the state weighting matrix $Q(k)=I_{4}$, the state final-value weighting matrix $Q(T)=I_{4}$, and the control weighting matrix $R(k)=$ $I_{4}$. We take the initial state as $x(0)=\left[\begin{array}{ll}46 & -32\end{array}\right]^{T}$ and set time horizon as $T=50$ in the simulation. According to Theorem 5 we obtain the optimal control sequence that minimizes the quadratic cost function in (8). The actuator assignment sequence is shown in Figure 2, where " 1 " and " 2 " in the $y$-axis denote the working modes. The data packet dropouts of channels 1 and 2 are shown in Figures 3 and 4, where " 1 " and " 0 " in the $y$-axis denote cases of the packet arrival and packet loss, respectively. The simulation result of the state trajectories is depicted in Figure 5, which has verified that our goal is achieved. Besides, we can clearly observe from Figure 5 that there are prominent variations in the state curves around the equilibrium state in the control stage, which means that the access constraints and packet dropouts have a larger effect on the dynamic performance of the networked systems.

\section{Conclusions}

This paper has investigated finite-time optimal controller design problem for networked control systems with network access constraints and packet dropouts. Since the presence of limited bandwidth network, there are limitations on the number of active actuators and packet dropouts between the controller and the actuators of NCSs. The channelaccess status of the actuators is governed by a group random

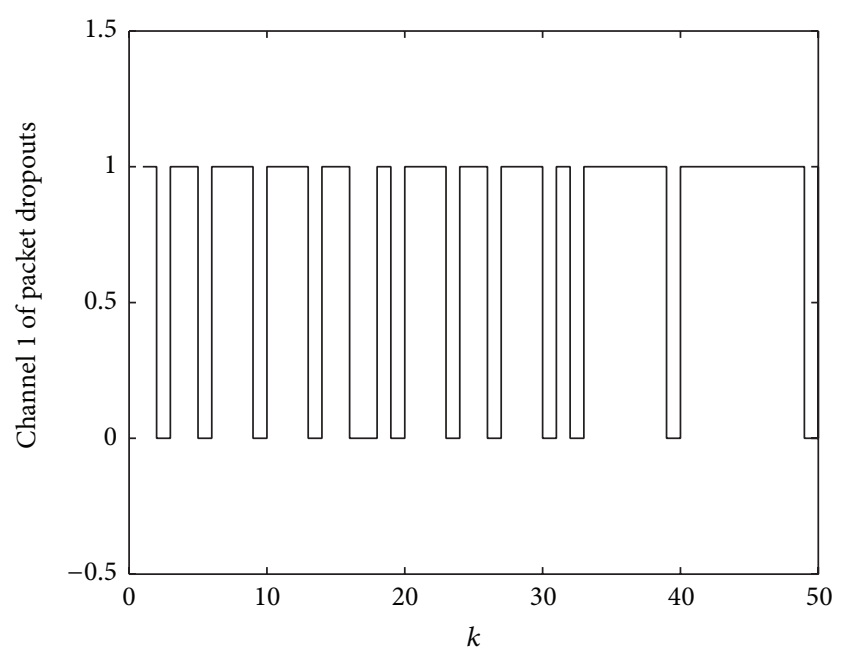

FIGURE 3: Channel 1 of data packet dropouts.

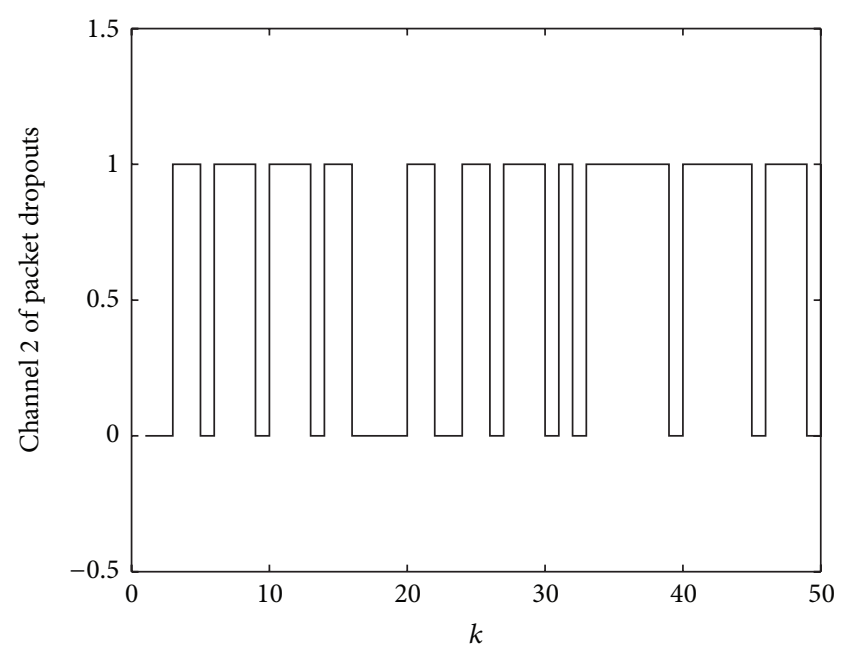

FIGURE 4: Channel 2 of data packet dropouts.

access protocol modeled as a Markov process, and packet dropouts are described by an iid Bernoulli process whose acknowledgement is always available to the controller. For such a system, an optimal control design methodology is addressed to satisfy the quadratic cost function by applying the well-developed theory for jump linear systems and stochastic optimal control while guaranteeing the meansquare exponential stability of networked control systems. Simulation results have demonstrated the feasibility and effectiveness of the addressed control scheme.

We assume that full-state information is available for measurements in this paper. In many cases, this assumption does not hold. Under such circumstances, we can perform a state estimation and then design an optimal state feedback controller. Furthermore, many systems may be subject to access constraints and pocket-loss at both sides of the communication network. Therefore, it is of great interest to seek for an effective controller design framework by taking 


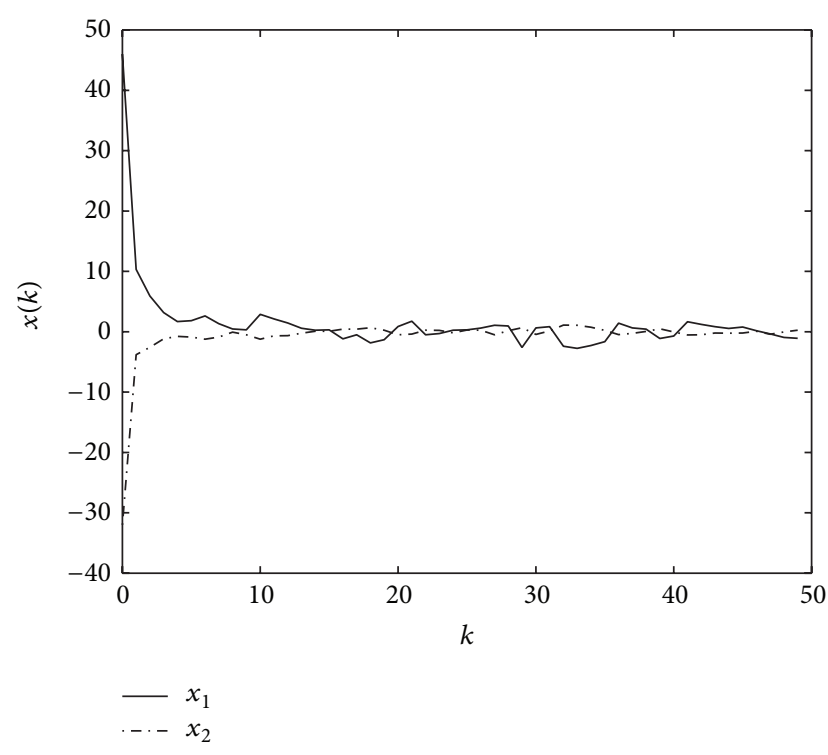

FIGURE 5: State trajectories.

into simultaneous consideration the constraints at both sides of the network.

\section{Conflict of Interests}

The authors declare that there is no conflict of interests regarding the publication of this paper.

\section{Acknowledgments}

This work was supported by the Natural Science Foundation of China under Grants nos. 61174060, 61273107, and 61263003, the Dalian Leading Talent Project under Grant no. 2012Z0036, Dalian, China, and the Fundamental Research Funds for the Central Universities under Grant no. 3132013334, China.

\section{References}

[1] S. Battilotti, "Control over a communication channel with random noise and delays," Automatica, vol. 44, no. 2, pp. 348-360, 2008.

[2] J. Hu, Z. Wang, B. Shen, and H. Gao, "Gain-constrained recursive filtering with stochastic nonlinearities and probabilistic sensor delays," IEEE Transactions on Signal Processing, vol. 61, no. 5, pp. 1230-1238, 2013.

[3] X. Jiang, Q. Han, S. Liu, and A. Xue, "A new $H_{\infty}$ stabilization criterion for networked control systems," IEEE Transactions on Automatic Control, vol. 53, no. 4, pp. 1025-1032, 2008.

[4] J. Hu, D. Chen, and J. Du, "State estimation for a class of discrete nonlinear systems with randomly occurring uncertainties and distributed sensor delays," International Journal of General Systems, vol. 43, no. 3-4, pp. 387-401, 2014.

[5] V. Gupta, B. Hassibi, and R. M. Murray, "Optimal LQG control across packet-dropping links," Systems and Control Letters, vol. 56, no. 6, pp. 439-446, 2007.
[6] J. Hu, Z. Wang, B. Shen, and H. Gao, "Quantised recursive filtering for a class of nonlinear systems with multiplicative noises and missing measurements," International Journal of Control, vol. 86, no. 4, pp. 650-663, 2013.

[7] W. Zhang and L. Yu, "Output feedback stabilization of networked control systems with packet dropouts," IEEE Transactions on Automatic Control, vol. 52, no. 9, pp. 1705-1710, 2007.

[8] J. Hu, Z. Wang, and H. Gao, "Recursive filtering with random parameter matrices, multiple fading measurements and correlated noises," Automatica, vol. 49, no. 11, pp. 3440-3448, 2013.

[9] N. Elia and S. K. Mitter, "Stabilization of linear systems with limited information," IEEE Transactions on Automatic Control, vol. 46, no. 9, pp. 1384-1400, 2001.

[10] S. Tatikonda and S. Mitter, "Control under communication constraints," IEEE Transactions on Automatic Control, vol. 49, no. 7, pp. 1056-1068, 2004.

[11] J. Hu, Z. Wang, H. Dong, and H. Gao, "Recent advances on recursive filtering and sliding mode design for networked nonlinear stochastic systems: a survey," Mathematical Problems in Engineering, vol. 2013, Article ID 646059, 12 pages, 2013.

[12] R. Brockett, "Stabilization of motor networks," in Proceedings of the 34th IEEE Conference on Decision and Control, pp. 14841488, New Orleans, La, USA, December 1995.

[13] M. S. Branicky, S. M. Phillips, and W. Zhang, "Scheduling and feedback co-design for networked control systems," in Proceedings of the 41st IEEE Conference on Decision and Control, pp. 1211-1217, Las Vegas, Nev, USA, December 2002.

[14] L. Zhang and D. Hristu-Varsakelis, "Communication and control co-design for networked control systems," Automatica, vol. 42, no. 6, pp. 953-958, 2006.

[15] G. Guo and H. Jin, "A switching system approach to actuator assignment with limited channels," International Journal of Robust and Nonlinear Control, vol. 20, no. 12, pp. 1407-1426, 2010.

[16] G. Guo, "A switching system approach to sensor and actuator assignment for stabilisation via limited multi-packet transmitting channels," International Journal of Control, vol. 84, no. 1, pp. 78-93, 2011.

[17] Z. Lu and G. Guo, "Communications and control co-design: a combined dynamic-static scheduling approach," Science China. Information Sciences, vol. 55, no. 11, pp. 2495-2507, 2012.

[18] M. E. M. Ben Gaid, A. Çela, and Y. Hamam, "Optimal integrated control and scheduling of systems with communication constraints," in Proceedings of the 44th IEEE Conference on Decision and Control, pp. 854-859, Seville, Spain, December 2005.

[19] B. Lincoln and B. Bernhardsson, "LQR optimization of linear system switching," IEEE Transactions on Automatic Control, vol. 47, no. 10, pp. 1701-1705, 2002.

[20] H. Rehbinder and M. Sanfridson, "Scheduling of a limited communication channel for optimal control," Automatica, vol. 40, no. 3, pp. 491-500, 2004.

[21] M. E. M. Ben Gaid, A. Çela, and Y. Hamam, "Optimal integrated control and scheduling of networked control systems with communication constraints: application to a car suspension system," IEEE Transactions on Control Systems Technology, vol. 14, no. 4, pp. 776-787, 2006.

[22] A. S. Matveev and A. V. Savkin, "The problem of LQG optimal control via a limited capacity communication channel," Systems and Control Letters, vol. 53, no. 1, pp. 51-64, 2004.

[23] F. A. Tobagi, "Multiaccess protocols in packet communication systems," IEEE Transactions on Communications, vol. 28, no. 4, pp. 468-488, 1980. 
[24] L. Y. Wang and G. Guo, "Control with random access protocol and packet dropouts," International Journal of Systems Science. Accepted.

[25] A. P. C. Gonçalves, A. R. Fioravanti, and J. C. Geromel, "Markov jump linear systems and filtering through network transmitted measurements," Signal Processing, vol. 90, no. 10, pp. 2842-2850, 2010.

[26] H. Ishii, " $H_{\infty}$ control with limited communication and message losses," Systems and Control Letters, vol. 57, no. 4, pp. 322-331, 2008.

[27] G. Guo, "Linear systems with medium-access constraint and Markov actuator assignment," IEEE Transactions on Circuits and Systems I: Regular Papers, vol. 57, no. 11, pp. 2999-3010, 2010.

[28] G. Guo and Z. B. Lu, "Control with Markov sensors/actuators assignment," IEEE Transactions on Automatic Control, vol. 57, no. 7, pp. 1799-1804, 2012.

[29] E. Yaz, "Control of randomly varying systems with prescribed degree of stability," IEEE Transactions on Automatic Control, vol. 33, no. 4, pp. 407-410, 1988. 


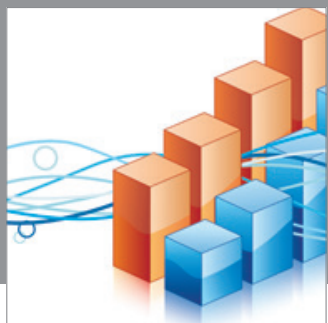

Advances in

Operations Research

mansans

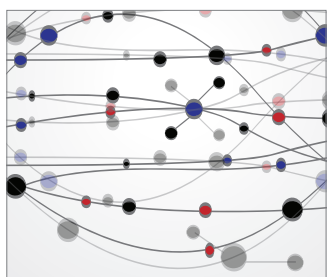

The Scientific World Journal
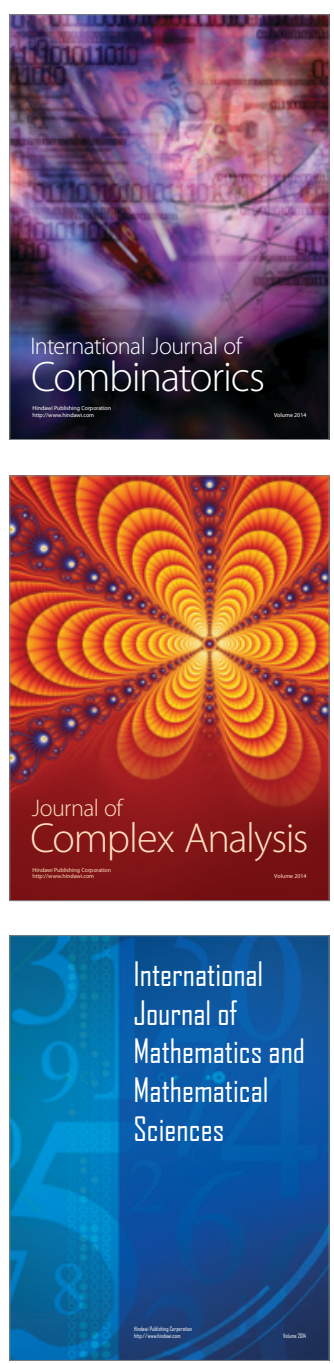
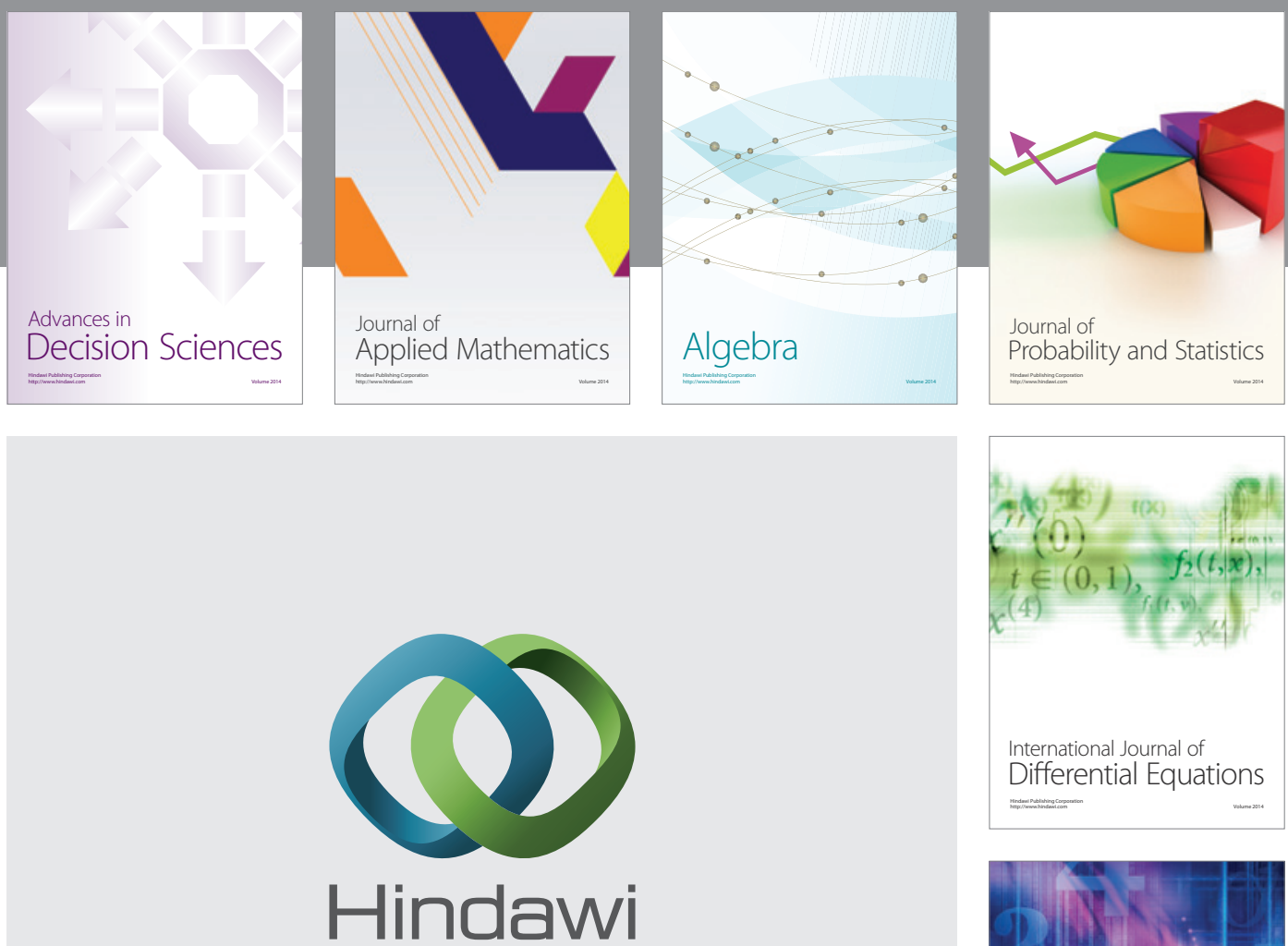

Submit your manuscripts at http://www.hindawi.com
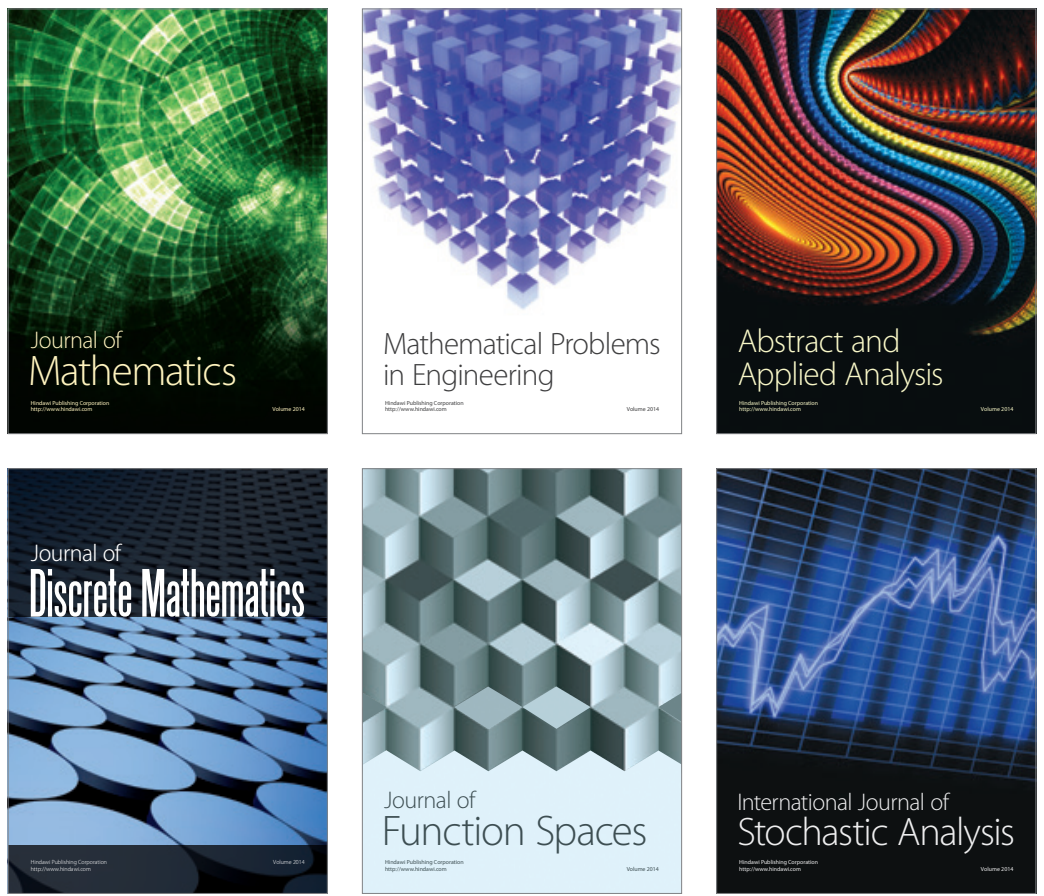

Journal of

Function Spaces

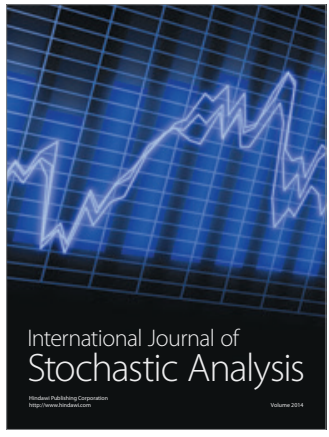

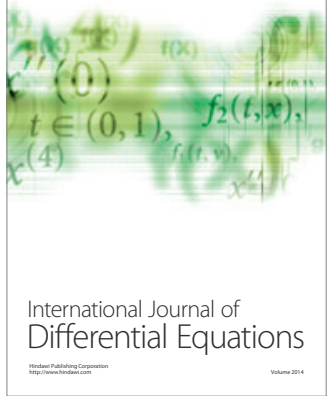
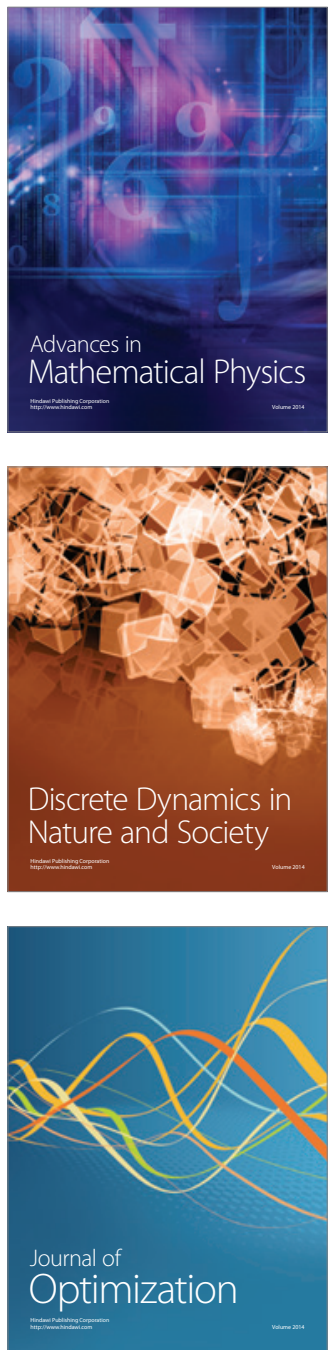